\title{
Impact of Muscle Mass as a Prognostic Factor for Failed Waiting Time Prior to Heart Transplantation
}

\author{
Luise Roehrich ${ }^{1,2,3 *}$, Simon H. Suendermann ${ }^{1,2,4}$, Isabell Anna Just ${ }^{1}$, \\ Laurenz Kopp Fernandes ${ }^{1}$, Jessica Schnettler ${ }^{1}$, Sebastian Kelle ${ }^{2,5}$, Natalia Solowjowa ${ }^{1}$, \\ Julia Stein ${ }^{1}$, Manfred Hummel ${ }^{6}$, Jan Knierim ${ }^{1}$, Evgenij Potapov ${ }^{1,2}$, Christoph Knosalla ${ }^{1,2}$, \\ Volkmar Falk ${ }^{1,2,4,7}$ and Felix Schoenrath ${ }^{1,2}$

\begin{abstract}
${ }^{1}$ Department of Cardiothoracic and Vascular Surgery, German Heart Center Berlin, Berlin, Germany, ${ }^{2}$ German Centre for Cardiovascular Research (DZHK), Partner Site Berlin, Berlin, Germany, ${ }^{3}$ German Heart Foundation, Frankfurt am Main, Germany, ${ }^{4}$ Department of Cardiovascular Surgery, Charité-Universitätsmedizin Berlin, Corporate Member of Freie Universität Berlin and Humboldt-Universität zu Berlin, Berlin, Germany, ${ }^{5}$ Department of Internal Medicine, Cardiology, German Heart Center Berlin, Charité-Universitätsmedizin Berlin, Berlin, Germany, ${ }^{6}$ Paulinen Hospital, Berlin, Germany, ${ }^{7}$ Department of Health Sciences and Technology, ETH Zürich, Zurich, Switzerland
\end{abstract}

\section{OPEN ACCESS}

Edited by:

Andrew Richard Kolodziej, University of Kentucky, United States

Reviewed by:

Suguru Ohira,

Westchester Medical Center

United States

Kenichi Hongo,

Jikei University School of

Medicine, Japan

*Correspondence:

Luise Roehrich

roehrich@dhzb.de

Specialty section:

This article was submitted to Heart Failure and Transplantation,

a section of the journal

Frontiers in Cardiovascular Medicine

Received: 26 June 2021 Accepted: 13 September 2021

Published: 18 October 2021

Citation:

Roehrich L, Suendermann SH, Just IA,

Kopp Fernandes L, Schnettler J,

Kelle S, Solowjowa N, Stein J,

Hummel M, Knierim J, Potapov E, Knosalla C, Falk V and Schoenrath F (2021) Impact of Muscle Mass as a Prognostic Factor for Failed Waiting Time Prior to Heart Transplantation. Front. Cardiovasc. Med. 8:731293.

doi: 10.3389/fcvm.2021.731293
Objectives: Clinical deterioration during the waiting time impairs the prognosis of patients listed for heart transplantation. Reduced muscle mass increases the risk for mortality after cardiac surgery, but its impact on resilience against deterioration during the waiting time remains unclear.

Methods: We retrospectively analyzed data from 93 patients without a VAD who were listed in Eurotransplant status "high urgent (HU)" for heart transplantation between January 2015 and October 2020. The axial muscle area of the erector spinae muscles at the level of thoracic vertebra 12 indexed to body surface area (TMESA/BSA) measured in the preoperative thoracic computed tomography scan was used to measure muscle mass.

Results: Forty patients (43\%) underwent emergency VAD implantation during the waiting time and four patients (4\%) died during the waiting time. The risk of emergency VAD implantation/death during the waiting time decreased by $10 \%$ for every $\mathrm{cm}^{2} / \mathrm{m}^{2}$ increase in muscle area [OR 0.901 (95\% Cl: 0.808-0.996); $p=0.049]$. After adjusting for gender [OR 0.318 (95\% Cl: 0.087-1.073); $p=0.072$ ], mean pulmonary artery pressure [OR 1.061 (95\% Cl: 0.999-1.131); $p=0.060$ ], C-reactive protein [OR 1.352 (95\% Cl: 0.986-2.027); $p=0.096$ ], and hemoglobin [OR 0.862 (95\% Cl: 0.618-1.177); $p=0.360$ ], TMESA/BSA [OR 0.815 (95\% Cl: 0.698-0.936); $p=0.006$ ] remained an independent risk factor for emergency $V A D$ implantation/death during the $\mathrm{HU}$ waiting time.

Conclusion: Muscle area of the erector spinae muscle appears to be a potential, easily identifiable risk factor for emergency VAD implantation or death in patients on the $\mathrm{HU}$ waiting list for heart transplantation. Identifying patients at risk could help optimize the outcome and the timing of VAD support.

Keywords: waiting list mortality, heart transplantation, ventricular assist device, sarcopenia, frailty 


\section{INTRODUCTION}

Cardiac transplantation remains the gold standard for end-stage heart failure, but donor organ shortage causes long waiting times even for critically ill patients in the Eurotransplant region $(1,2)$. In 2019, 956 patients were removed from the Eurotransplant waiting list, 665 patients underwent transplantation (3), and 244 were delisted due to death, being unfit for transplantation or other reasons (3); 159 patients died during the waiting time, and 39 patients were deemed ineligible for transplantation (3).

Several risk factors for waiting list mortality were identified, including impaired renal function and low serum albumin (4). Frailty, the reduced resilience against stressors (5), and sarcopenia, reduced muscle mass and function (6), appear to be relevant risk factors for waiting list mortality in patients undergoing kidney $(7,8)$, lung (9), or liver transplantation $(10,11)$. To the best of our knowledge, this is the first study investigating the impact of muscle mass on the prognosis during the waiting time of adult patients with a "high urgent (HU)" Eurotransplant status for heart transplantation (comparable to UNOS status 3) $(12,13)$.

\section{MATERIALS AND METHODS}

\section{Study Design}

From January 2015 to October 2020, 161 adult patients were granted the HU status for heart transplantation at the German Heart Center Berlin; 68 (42\%) patients were already supported with a ventricular assist device (VAD) at the time of the first request for $\mathrm{HU}$ listing and were excluded from further analysis. Pediatric patients $<16$ years, patients listed for combined organ transplantation, and patients who were delisted due to stabilization did not qualify for this study. Of the remaining 93 patients, 40 (43\%) underwent emergency VAD implantation and 4 (4\%) died during the HU waiting time (Figure 1); consequently, $49(53 \%)$ patients reached transplantation and $44(47 \%)$, the outcome of interest. Baseline characteristics were collected at the time of first listing in the HU status. Organs were allocated in accordance with the Eurotransplant guidelines (14). The time of emergency VAD implantation was selected according to an intrahospital standard operating procedure of daily clinical reevaluation (Figure 2).

This study was approved by the Ethics Committee of CharitéUniversitätsmedizin Berlin (EA2/236/17).

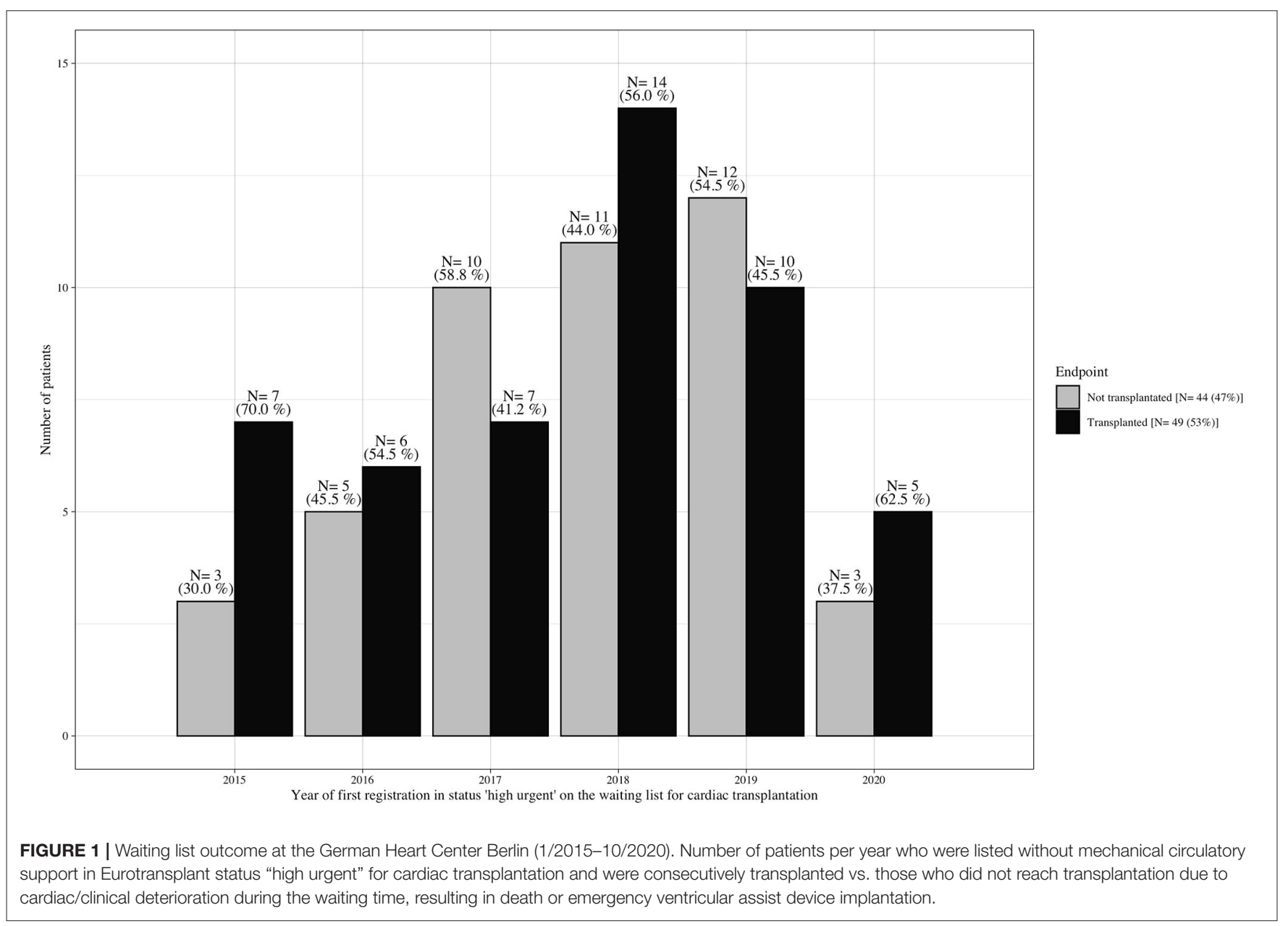




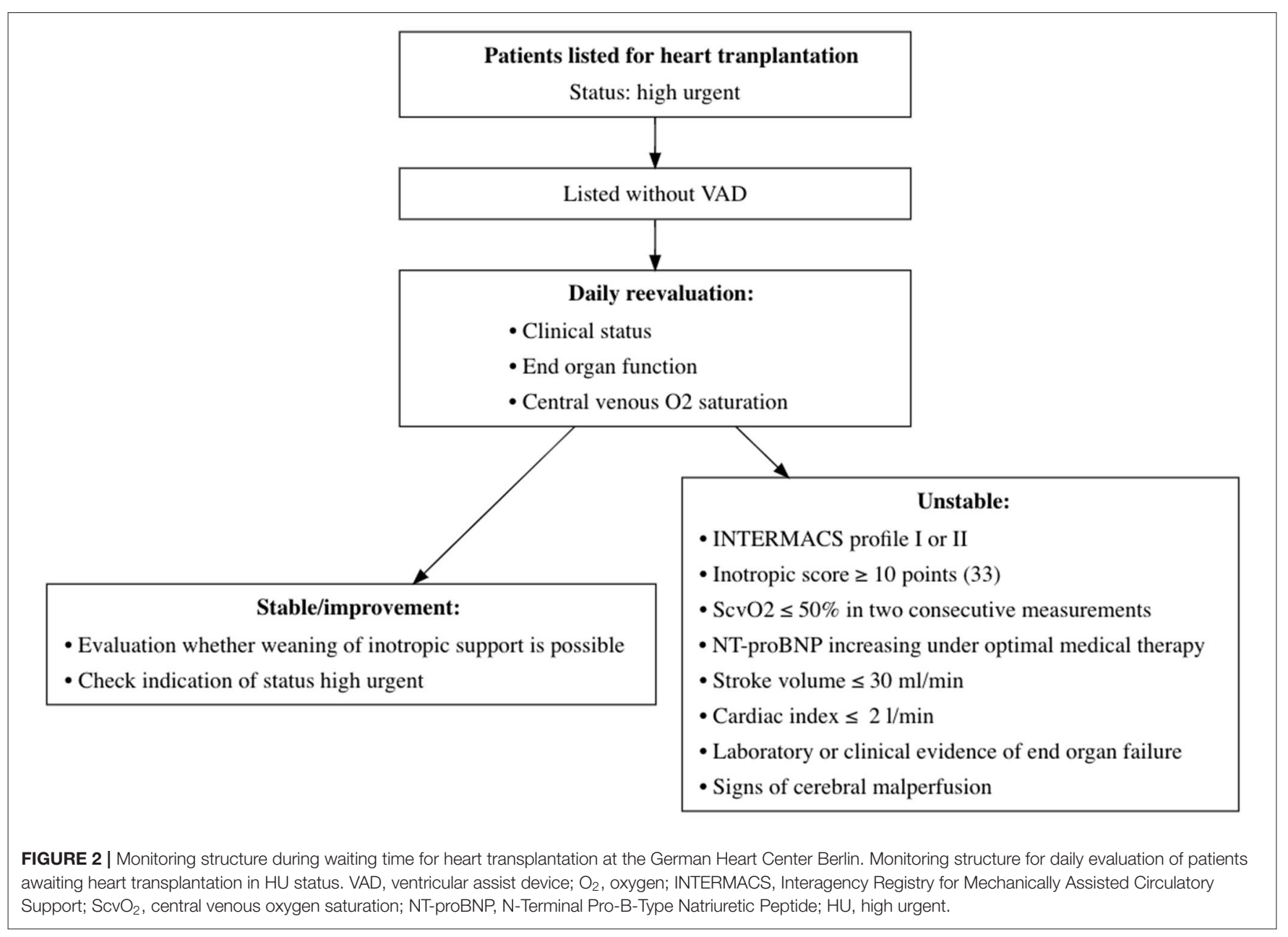

\section{Imaging}

To measure muscle mass, the thoracic computed tomography (CT) scan used for the surgical planning and for excluding infection or malignancies during the evaluation process was analyzed with regard to the erector spinae muscles at level Th12 (TMESA) in a single axial image indexed to the body surface area (BSA) estimated with the DuBois formula $(15,16)$ (Figure 3). Measuring the muscle area in CT scans is considered a gold standard for evaluating muscle mass $(6,17)$. Wang et al. demonstrated high reliability of image-based estimations of the muscle area (18). The predictive value of the area of the erector spinae muscles was shown in several clinical relations $(15,19,20)$. Referring to the results of Minegishi et al., we defined reduced muscle mass as a TMESA/BSA $\leq 17.2 \mathrm{~cm}^{2} / \mathrm{m}^{2}(15)$.

\section{Statistical Method}

Ordinally and nominally scaled parameters are described in absolute (\#) and relative (\%) terms; between-group comparisons were performed using the $\chi^{2}$-test. After testing for a Gaussian distribution, metric values were analyzed using Student's $t$-test or the Mann-Whitney $U$-test, as appropriate. For normally distributed values, the mean value with standard deviation is stated; for other distributions, the median is indicated with the first and third quartile.

Clinically relevant risk factors were examined using univariable logistic regression analysis from which odds ratios (OR) and 95\% confidence intervals were calculated. Factors with a $p<0.1$ in the univariable analysis and gender were included in a multivariable approach, and the best fitting model according to the Akaike Information Criterion was chosen.

Cumulative incidences of death/VAD and transplantation are displayed graphically. Event-free survival during the waiting time (events defined as death and/or emergency VAD implantation) was estimated with a Kaplan-Meier analysis with the time of listing as the starting point. Patients undergoing cardiac transplantation were censored.

All $p$-values should be read descriptively.

\section{RESULTS}

\section{Baseline Characteristics}

The 49 patients (53\%) who underwent transplantation spent 89 days on the HU waiting list; in comparison, patients who did not reach primary transplantation $(n=44 ; 47 \%)$ spent 36 days on the waiting list [ $89 \mathrm{~d}$ (IQR 40-130) vs. $35.5 \mathrm{~d}$ (IQR 15-93); 

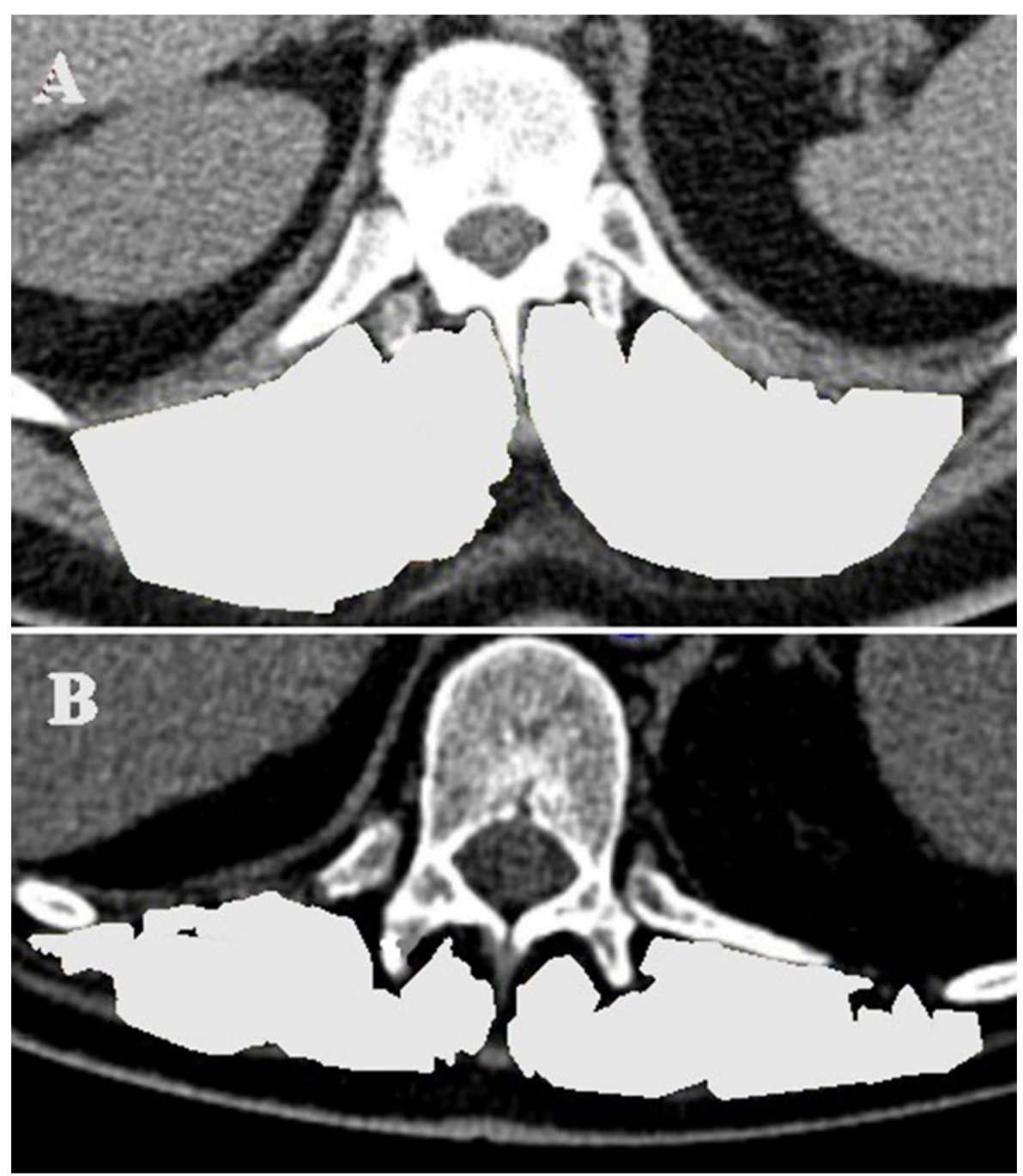

FIGURE 3 | TMESA measurement in an axial CT image at level of the 12th thoracic vertebrae (TMESA area marked in light gray). (A) Male patient without impaired muscle area. (B) Male patient with impaired muscle area. TMESA, total area of the erector spinae muscles; CT, computed tomography.

$p=0.007]$. The course of the HU waiting time in this cohort is displayed in Figure 4. The groups did not differ in allocationrelevant parameters such as weight, height, gender, age, or blood type. No difference was observed in secondary organ function represented by the glomerular filtration rate (eGFR) calculated with the Modification of Diet in Renal Disease (MDRD) formula and the Model For End-Stage Liver Disease XI (MELD-XI) score (21) (Table 1).

Thirty-nine (42\%) of the patients had a TMESA/BSA $\leq 17.2$ $\mathrm{cm}^{2} / \mathrm{m}^{2}$ [compare Minegishi et al. (15)] and were therefore defined as reduced muscle mass. The impact of muscle mass appeared to increase if the waiting time exceeded 20 days (Figure 5). Patients with a significantly lower TMESA/BSA had a lower creatinine level and MELD-XI score and a higher cardiac index (Table 2).

In the vast majority of the patients $[N=36(81 \%)]$, VAD implantation was indicated due to cardiogenic shock despite high-dose combined inotropic support after exhausting conservative treatment options; therefore, by definition, these patients were unstable [Interagency Registry for Mechanically Assisted Circulatory Support (INTERMACS) profile 1 or 2] prior to implantation. In 11 (25\%) patients, cardiac decompensation was triggered by a systemic infection. The 12-month survival after surgery was $85 \%$ (95\% CI: $0.75-0.96 \%)$ in transplanted patients and 74\% (95\% CI: $0.61-0.89 \%)$ after emergency VAD implantation. The further course of treatment is displayed in Figure 6.

Four patients died during the waiting time: all underwent short-term circulatory support implantation [three extracorporeal membrane oxygenation (ECMO) and one Impella] in INTERMACS profile 1. One patient required mechanical cardiopulmonary resuscitation due to cardiogenic shock; three patients developed combined cardiogenic and septic shock. 


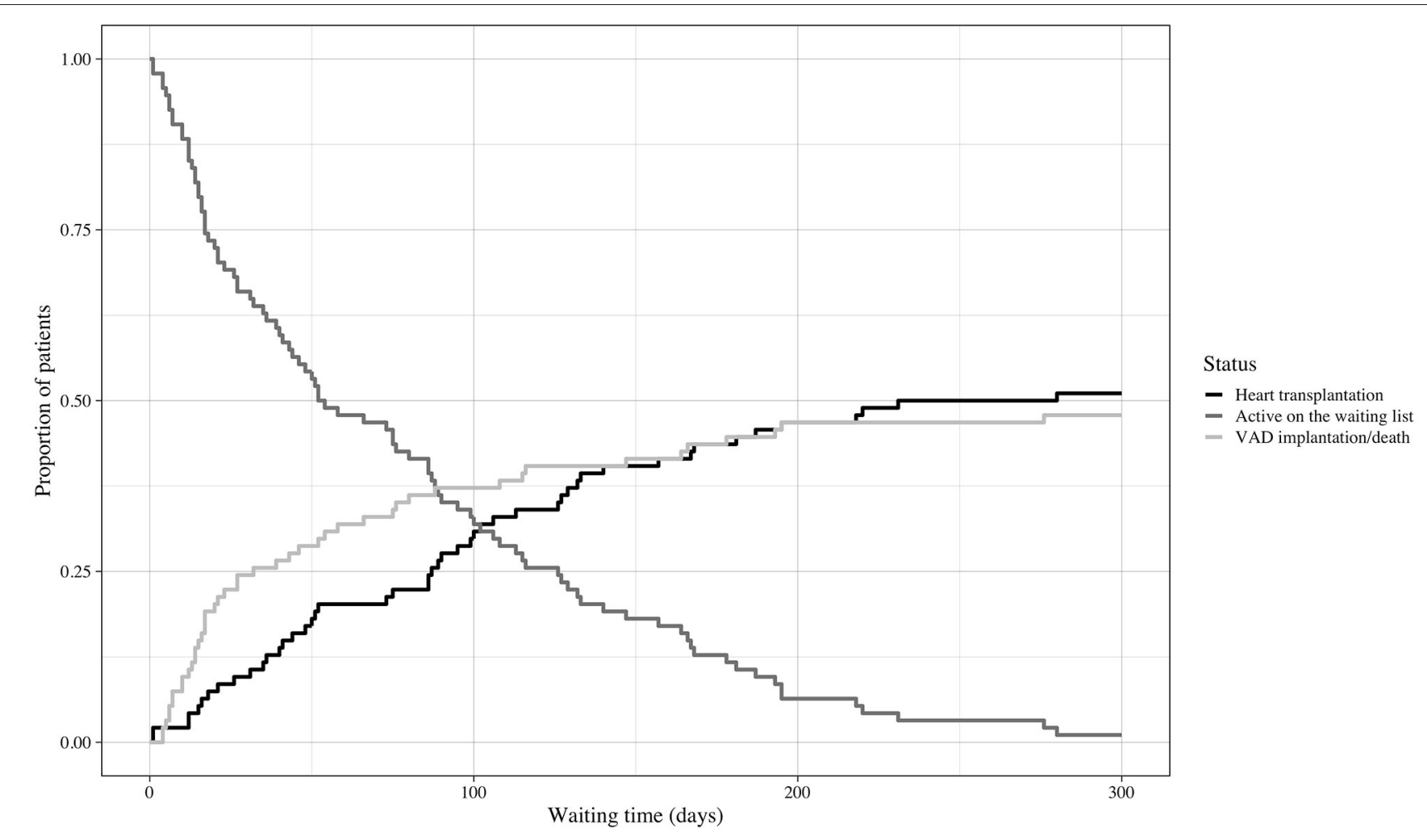

FIGURE 4 | Course of waiting time. Cumulative incidences and course of waiting time. Event: heart transplantation or emergency VAD implantation/death during the HU waiting time. VAD, ventricular assist device; HU, high urgent.

\section{Impact of Muscle Mass}

Patients who underwent emergency VAD implantation or died during the HU waiting time had a lower TMESA/BSA (Table 1). The univariate logistic analysis revealed a significantly decreased risk of emergency VAD implantation or death during the $\mathrm{HU}$ waiting time by $10 \%$ for every $\mathrm{cm}^{2} / \mathrm{m}^{2}$ increase in TMESA/BSA [OR 0.901 (95\% CI: 0.808-0.996); $p=0.049$, compare Figure 7]. Further risk factors are displayed in Figure 7. Multivariable analysis adjusted for gender, mean PAP, CRP, and hemoglobin levels revealed muscle mass as an independent risk factor for emergency VAD implantation or death, whereas the other parameters were not significant in this model (Figure 8).

\section{DISCUSSION}

\section{Impact of Muscle Mass on the Prognosis During the Waiting Time}

If the hemodynamic situation deteriorates during the $\mathrm{HU}$ waiting time and no suitable donor organ is available, mechanical circulatory support remains the only therapeutic option. Close monitoring for a further decline in cardiac function while waiting for heart transplantation is crucial. If patients met certain criteria, they underwent emergency VAD implantation according to our institutional guidelines (Figure 2).
Muscle mass, represented by TMESA/BSA, was identified as a possible risk factor for emergency VAD implantation or death during the HU waiting time for heart transplantation. It might indicate a reduced resilience against stressors, such as infections or hemodynamic challenges with the need to escalate positive inotropic therapy. Comparable results were published for the impact of frailty, of which sarcopenia can be a symptom, prior to kidney, liver, and lung transplantation, with an increased waiting list mortality for frail and/or sarcopenic patients (7-11). Mostly, a (modified) Fried Frailty Phenotype was used to identify frailty and showed its prognostic value for waiting list mortality irrespective of disease-specific risk factors (5).

Minegishi et al. demonstrated the impact of a reduced TMESA/BSA $\leq 17.2 \mathrm{~cm}^{2} / \mathrm{m}^{2}$ on the outcome after pneumonia (15). Referring to their results, reduced muscle mass was defined by TMESA/BSA $\leq 17.2 \mathrm{~cm}^{2} / \mathrm{m}^{2}$. TMESA/BSA $\leq 17.2 \mathrm{~cm}^{2} / \mathrm{m}^{2}$ was a relevant risk factor for emergency VAD implantation or death during the HU waiting time in advanced heart failure patients. Its impact was independent of gender and other known risk factors such as congestion as an early sign of beginning cardiac deterioration (represented by mean PAP), inflammation (represented by CRP), and anemia (represented by hemoglobin levels). With $42 \%$, the prevalence of reduced muscle mass in our cohort is comparable to the expected prevalence of frailty in this cohort, which is around $45 \%$ according to the 2019 position paper on frailty in heart failure patients and the estimated frailty rate 
TABLE 1 | Baseline characteristics at time of waiting list registration in status "high urgent."

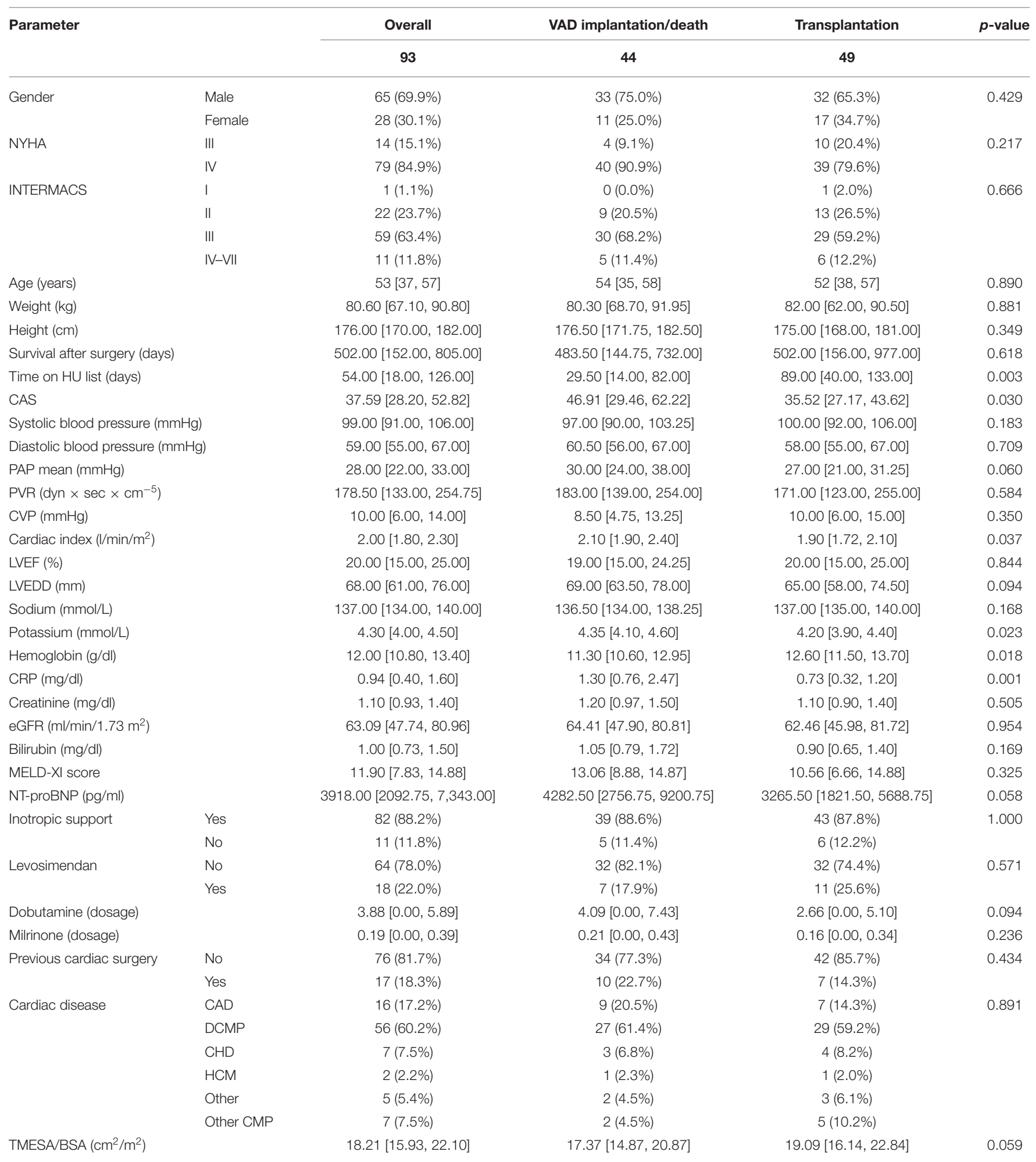

CAS, cardiac allocation score; PAP, pulmonary artery pressure; PVR, pulmonary vascular resistance; CVP, central venous pressure; LVEF, left ventricular ejection fraction; LVEDD, left ventricular end-diastolic volume; CRP, C-reactive protein; eGFR, estimated glomerular filtration rate; CAD, coronary artery disease; DCMP, dilated cardiomyopathy; CHD, congenital heart disease; HCM, hypertrophic cardiomyopathy; CMP, cardiomyopathy.

Data are presented as numbers (\%), mean (standard deviation), or median [25th quartile, 75th quartile] as appropriate and groups were compared using the Mann-Whitney U test or chi-square test as appropriate. 


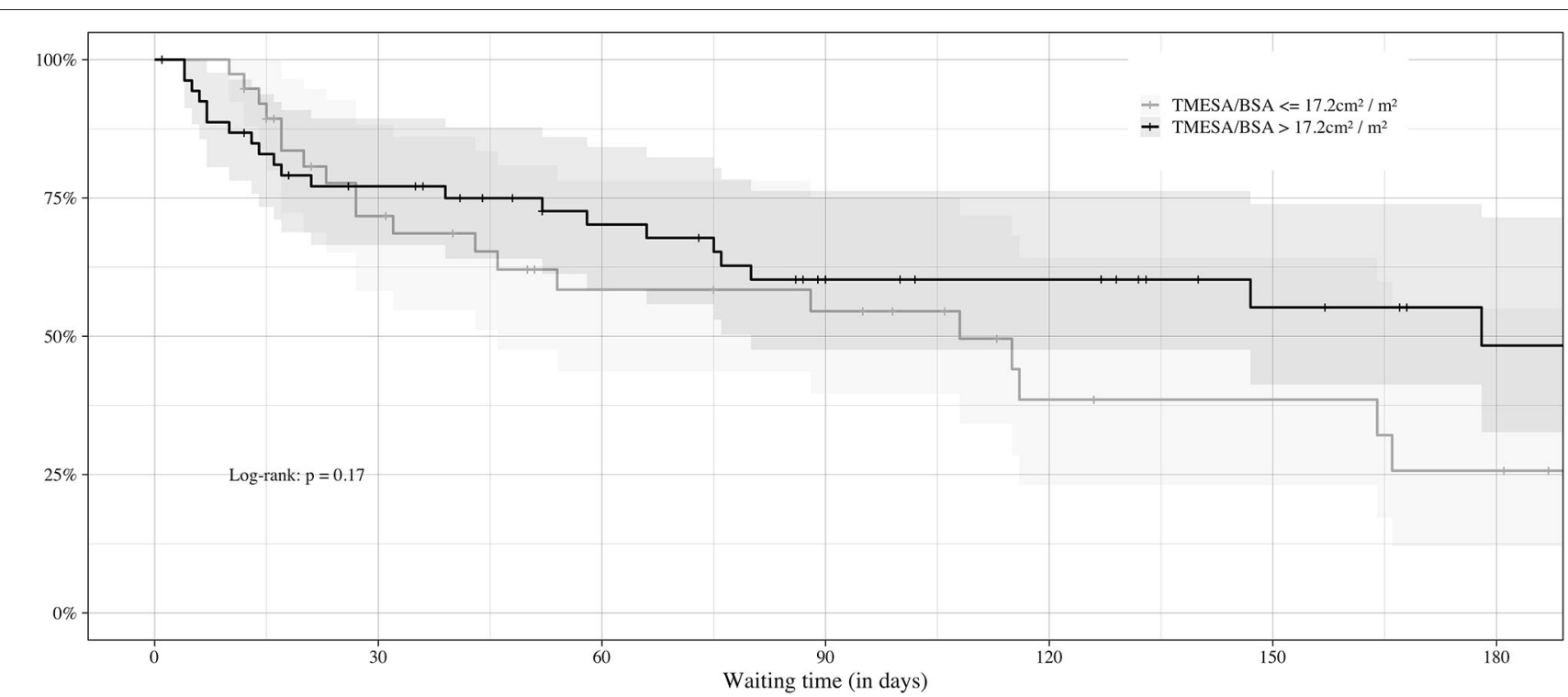

Number at risk: $\mathrm{n}(\%)$

\begin{tabular}{|c|c|c|c|c|c|c|}
\hline $39(100)$ & $24(62)$ & $16(41)$ & $14(36)$ & $7(18)$ & \\
\hline$-54(100)$ & $38(70)$ & $29(54)$ & $20(37)$ & $17(31)$ & $11(10)$ \\
\hline 0 & 30 & 60 & Waiting time (in days) & 120 \\
\hline
\end{tabular}

FIGURE 5 | Prognosis of waiting time in regard of sarcopenia. Impact of muscle mass on the course of HU waiting time. Event: death or emergency VAD implantation during the HU waiting time, censored: heart transplantation. VAD, ventricular assist device; TMESA/BSA, total area of the erector spinae muscles/body surface area; $\mathrm{HU}$, high urgent.

of $44.5 \%$ in the meta-analysis of Denfeld et al. (22) and Vitale et al. (23).

The impact of reduced muscle mass increases the longer the waiting time is. These findings are in line with our pathophysiological consideration that, in the early phase of waiting, muscle mass and sarcopenia play a less important role compared with deteriorating hemodynamic stability despite inotropic support at the time of listing $(4,24)$. Our impression is that, unlike in patients awaiting kidney, liver, or lung transplantation, the organ-specific hemodynamic risk of progressing cardiac instability in patients with end-stage heart failure may overlay the potential vulnerability due to reduced muscle mass, especially during the initial period after listing in HU status. Additionally, patients with a reduced muscle mass had a slightly better cardiac index at baseline, giving these patients a possible starting advantage compensating the effect of muscle mass in this early phase. Creatinine levels and MELD-XI scores were lower in the cohort with TMESA/BSA $\leq 17.2 \mathrm{~cm}^{2} / \mathrm{m}^{2}$, possibly owing to lower creatinine levels due to decreased muscle mass.

Adapting the evaluation of muscle mass to the standard monitoring protocol could contribute to identifying patients at risk of decompensation and optimizing the timing of VAD implantation if deterioration occurs.

\section{CT-Guided Measurement of the Muscle Mass}

TMESA/BSA was shown to be an effective method for evaluating muscle mass in these patients, being measurable in the standard thoracic CT scan prior to listing, which is needed in most patients for evaluation, even in emergency situations. In contrast, the iliopsoas muscle, which is widely researched for an evaluation of muscle area (6), would require an additional CT scan of the abdomen, which may not be standard prior to thoracic surgery. Additionally, being a minor muscle, the iliopsoas muscle may not be as representative as other core muscles (6).

Gold standards for diagnosing muscle mass, sarcopenia and frailty in advanced heart failure patients are yet to be defined. One of the most commonly used methods to evaluate frailty is the Fried Frailty Phenotype, which includes with gait speed, grip strength, and weight loss several components of sarcopenia (5). The evaluation of frailty in patients referred for heart transplantation was described by Leng et al. (25) who found an increased post-transplant mortality in frail patients.

However, in patients on inotropic support waiting in the "high urgent" status and/or in UNOS status 3, these assessments lack feasibility, because these patients by definition suffer from severe exercise intolerance or severe ventricular arrhythmias, both of which preclude the estimation of physical performance, 
TABLE 2 | Baseline characteristics at time of waiting list registration in status "high urgent": patients with reduced muscle area vs. patients with TMESA/BSA $>17.2 \mathrm{~cm}^{2} / \mathrm{m}^{2}$.

\begin{tabular}{|c|c|c|c|c|c|}
\hline \multirow[t]{2}{*}{ Parameter } & & Overall & TMESA/BSA $\leq 17.2 \mathrm{~cm}^{2} / \mathrm{m}^{2}$ & TMESA/BSA $>17.2 \mathrm{~cm}^{2} / \mathrm{m}^{2}$ & \multirow[t]{2}{*}{$p$-value } \\
\hline & & 93 & 39 & 54 & \\
\hline \multirow[t]{2}{*}{ Gender } & Male & 65 (69.9\%) & $17(43.6 \%)$ & $48(88.9 \%)$ & $<0.001$ \\
\hline & Female & $28(30.1 \%)$ & $22(56.4 \%)$ & $6(11.1 \%)$ & \\
\hline \multirow[t]{2}{*}{ NYHA } & III & $14(15.1 \%)$ & $4(10.3 \%)$ & 10 (18.5\%) & 0.420 \\
\hline & IV & 79 (84.9\%) & 35 (89.7\%) & 44 (81.5\%) & \\
\hline \multirow[t]{4}{*}{ INTERMACS } & 1 & $1(1.1 \%)$ & $1(2.6 \%)$ & $0(0.0 \%)$ & 0.866 \\
\hline & $\|$ & $22(23.7 \%)$ & $10(25.6 \%)$ & $12(22.2 \%)$ & \\
\hline & III & $59(63.4 \%)$ & $23(59.0 \%)$ & $36(66.7 \%)$ & \\
\hline & $\mid \mathrm{V}-\mathrm{VII}$ & $11(11.8 \%)$ & $5(12.8 \%)$ & $6(11.1 \%)$ & \\
\hline Age (years) & & $53.00[37,57]$ & $52.00[34,57]$ & $54.00[44,57]$ & 0.320 \\
\hline PAP mean (mmHg) & & $28.00[22.00,33.00]$ & $27.00[22.25,31.50]$ & $30.00[22.50,36.75]$ & 0.177 \\
\hline Cardiac index $\left(\mathrm{L} / \mathrm{min} / \mathrm{m}^{2}\right)$ & & $2.00[1.80,2.30]$ & $2.10[1.80,2.50]$ & $1.90[1.70,2.20]$ & 0.035 \\
\hline LVEF (\%) & & $20.00[15.00,25.00]$ & $20.00[15.00,22.75]$ & $19.50[15.00,25.00]$ & 0.412 \\
\hline LVEDD (mm) & & $6.80[6.10,7.60]$ & $6.70[5.80,7.55]$ & $6.85[6.23,7.68]$ & 0.380 \\
\hline Sodium (mmol/l) & & $137.00[134.00,140.00]$ & $136.00[133.00,139.00]$ & $137.00[135.25,140.00]$ & 0.036 \\
\hline Potassium (mmol/L) & & $4.30[4.00,4.50]$ & $4.30[3.90,4.50]$ & $4.30[4.03,4.50]$ & 0.534 \\
\hline Hemoglobin (g/dl) & & $12.00[10.80,13.40]$ & $11.70[10.65,13.65]$ & $12.30[10.93,13.20]$ & 0.621 \\
\hline CRP (mg/dl) & & $0.94[0.40,1.60]$ & $1.10[0.39,1.55]$ & $0.86[0.43,1.75]$ & 0.864 \\
\hline Creatinine (mg/dl) & & $1.10[0.93,1.40]$ & $1.00[0.80,1.35]$ & $1.25[0.98,1.50]$ & 0.022 \\
\hline eGFR (ml/min/1.73 m²) & & $63.09[47.74,80.96]$ & $69.47[47.98,85.67]$ & $62.43[46.13,77.91]$ & 0.346 \\
\hline Bilirubin (mg/dl) & & $1.00[0.73,1.50]$ & $1.10[0.70,1.45]$ & $0.97[0.74,1.67]$ & 0.791 \\
\hline MELD-XI score & & $11.90[7.83,14.88]$ & $9.57[6.84,13.25]$ & $13.23[8.64,15.14]$ & 0.038 \\
\hline NT-proBNP (pg/dl) & & 3918.00 [2092.75, 7343.00] & 3329.00 [2529.00, 9389.00] & 3967.00 [1829.00, 5871.00] & 0.441 \\
\hline \multirow[t]{6}{*}{ Cardiac disease } & CAD & $16(17.2 \%)$ & $5(12.8 \%)$ & $11(20.4 \%)$ & 0.176 \\
\hline & DCMP & $56(60.2 \%)$ & $22(56.4 \%)$ & 34 (63.0\%) & \\
\hline & $\mathrm{GUCH}$ & 7 (7.5\%) & $6(15.4 \%)$ & 1 (1.9\%) & \\
\hline & $\mathrm{HCM}$ & $2(2.2 \%)$ & $1(2.6 \%)$ & $1(1.9 \%)$ & \\
\hline & Other & 5 (5.4\%) & $3(7.7 \%)$ & 2 (3.7\%) & \\
\hline & Other CMP & 7 (7.5\%) & $2(5.1 \%)$ & 5 (9.3\%) & \\
\hline
\end{tabular}

CAS, cardiac allocation score; PAP, pulmonary artery pressure; LVEF, left ventricular ejection fraction; LVEDD, left ventricular end-diastolic volume; CRP, C-reactive protein; eGFR, estimated glomerular filtration rate; CAD, coronary artery disease; DCMP, dilated cardiomyopathy; CHD, congenital heart disease; HCM, hypertrophic cardiomyopathy; CMP, cardiomyopathy.

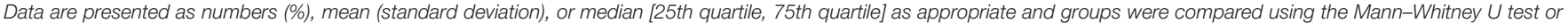
chi-square test as appropriate.

making the evaluation of muscle mass even more important. Additionally, results may be skewed by a floor effect due to exercise intolerance caused by heart failure, as described by the Heart Failure Association/European Society of Cardiology (23). This increases the importance of image-based methods for evaluating muscle area that are independent of patients' physical performance, although these methods represent only the muscle mass without considering functional aspect of sarcopenia.

For risk stratification prior to cardiac transplantation, muscle area as a surrogate for biological age could provide valuable information. It should be included in patients' routine evaluation, e.g., to identify patients who would benefit from early VAD implantation or well-defined prehabilitation programs, as will be assessed for instance in the upcoming trial of Stoppe et al., who are planning an interventional trial with a predefined nutritional and physical exercise program prior to VAD implantation in stable heart failure patients (26).

\section{Further Implications of Including Muscle Mass Measurement in Patients' Evaluation}

As sarcopenia is potentially reversible, both muscle mass and function could be positively influenced by following a healthy diet and starting a clinically adapted exercise program, or at least by engaging in passive movement support under close monitoring to prevent a further decline in physical reserve. In their review, McCann et al. identified several influenceable domains and showed the impact of preoperative rehabilitation prior to cardiac surgery on the improvement of the postoperative outcome (27). Developing a suitable intervention program for advanced heart failure patients could be the next step in optimizing the outcome while on the waiting list and after surgery, as suggested by Leng 


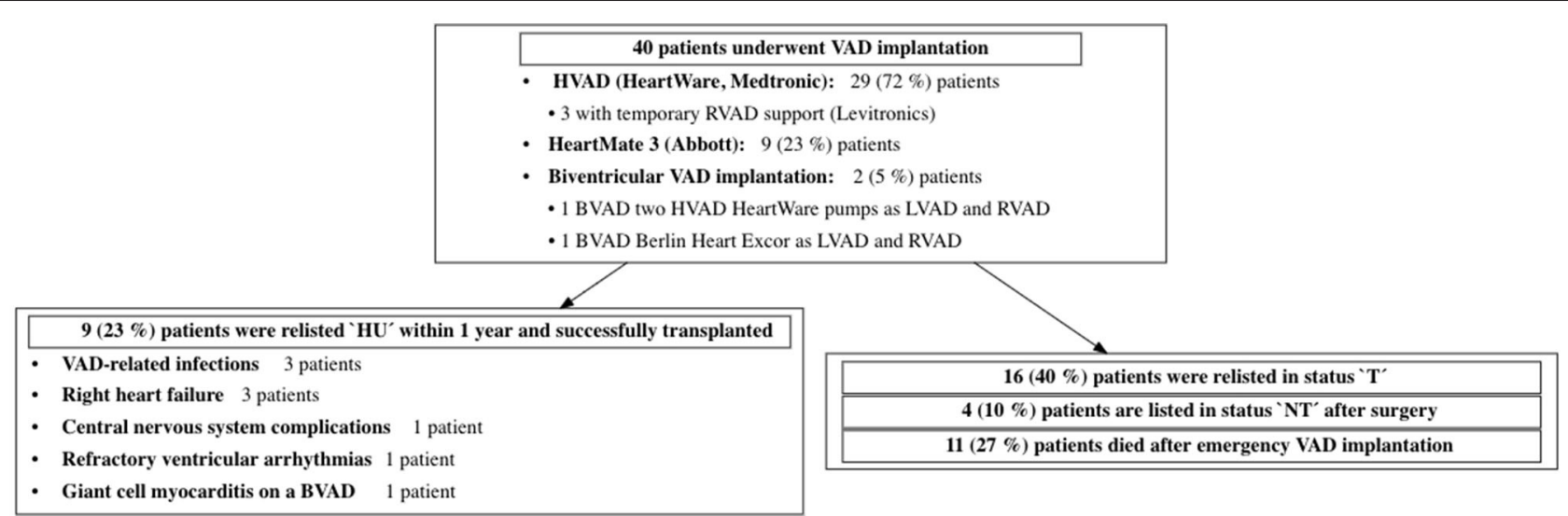

FIGURE 6 | Outcome after emergency VAD implantation during HU waiting time. VAD, ventricular assist device; LVAD, left ventricular assist device; RVAD, right ventricular assist device; BVAD, biventricular assist device; HU, 'high urgent'; T, 'transplantable'; NT, 'not transplantable.

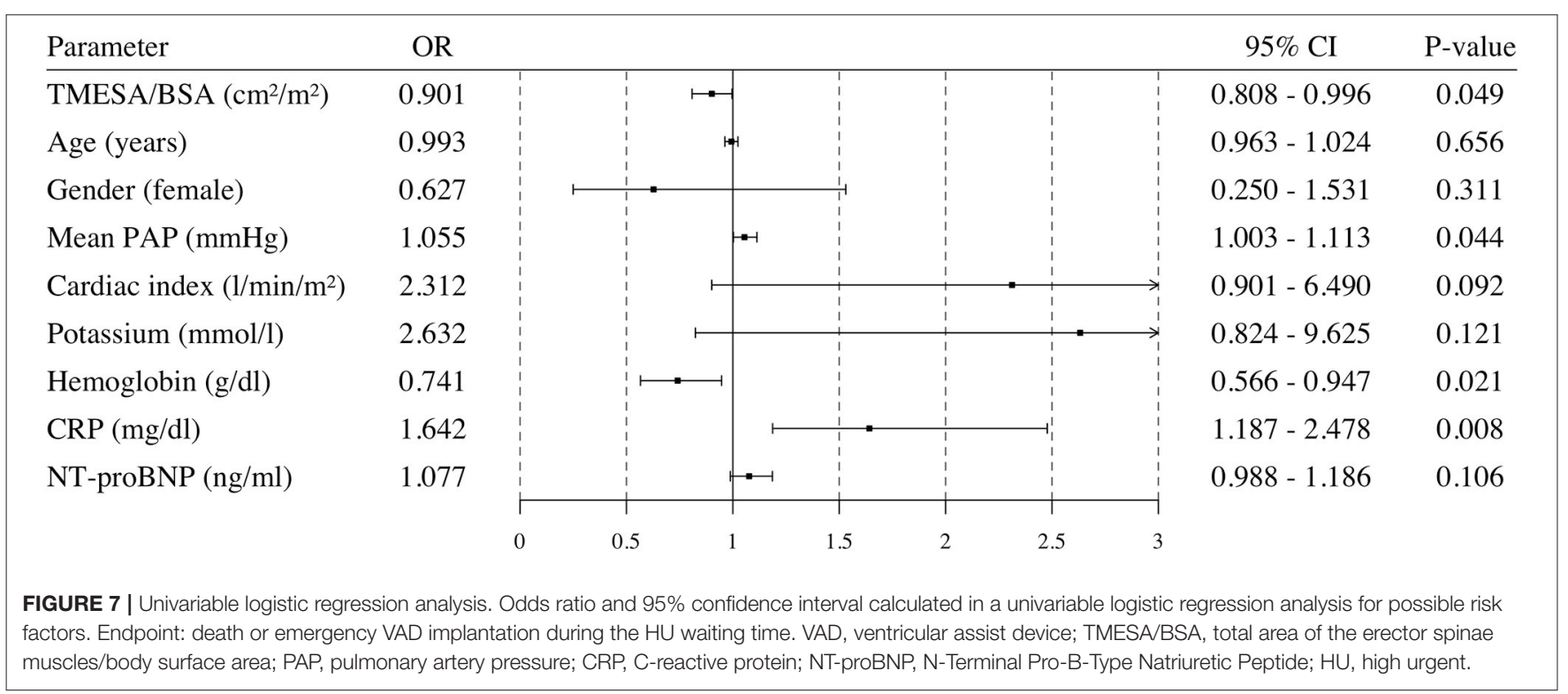

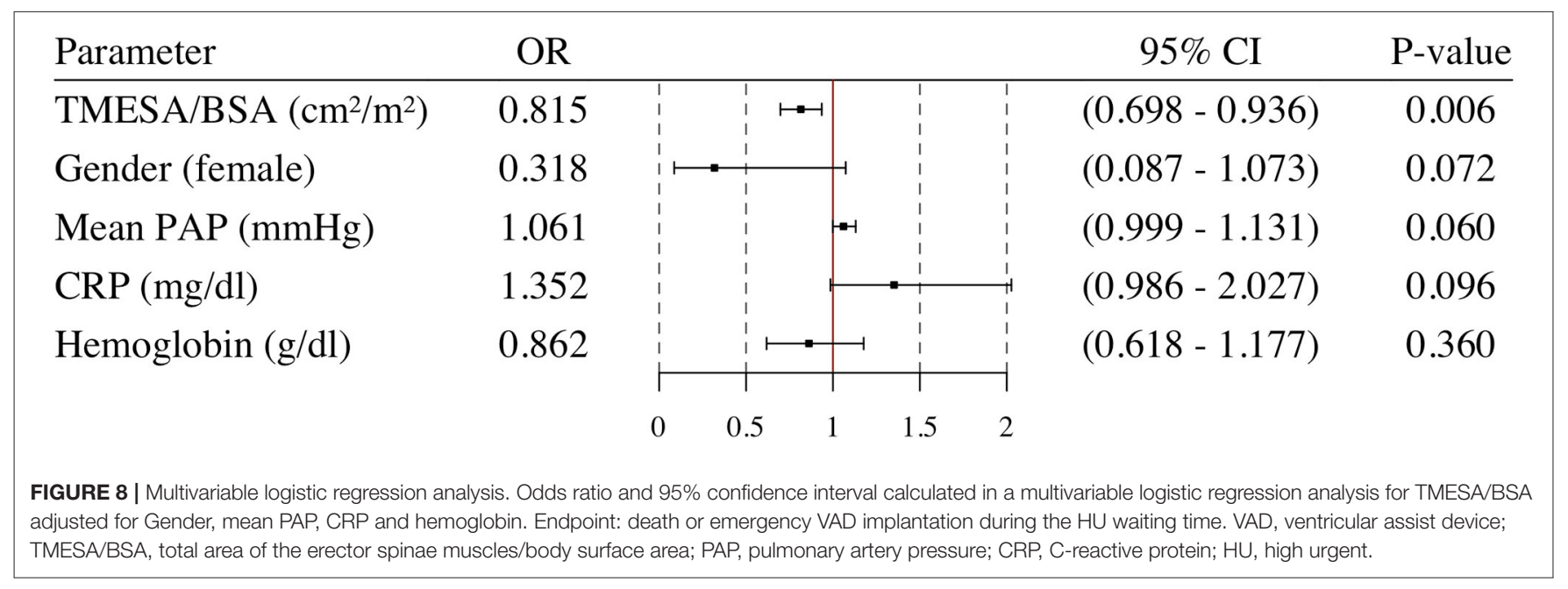


and Kittleson (25). Gimeno-Santos et al. were able to demonstrate the feasibility, safety, and potential benefit of a tailored exercise program during the waiting time for heart transplantation in 19 patients (28).

In some patients, VAD implantation could be a therapeutic option for disease-dependent sarcopenia, thereby optimizing the patient's status prior to cardiac transplantation. Maurer et al. and Jha et al. demonstrated the potential reversibility of frailty and therefore of impaired muscle function 2-6 months after VAD implantation without an exercise program when heart failure treatment was sufficient $(29,30)$. Physical training in particular should be safer and more efficient in VAD patients than in patients on inotropic support waiting without a VAD. The position paper of the European Society of Cardiology by Adamopoulos et al. highlights the benefits and limitations of exercise training in VAD patients (31). Although the surgery is more complex in patients on VAD support prior to heart transplantation, the outcome of patients with and without VAD implantation prior to cardiac transplantation is comparable (32).

\section{Possible Strategy Based on the Findings}

Muscle area evaluation seems to be a valuable tool for additional risk stratification. The standard evaluation process of advanced heart failure patients should include screening for reduced muscle mass.

Based on gender, weight, height, blood type, and availability of donor organs, the waiting time can be estimated and differs between regions. In patients with a reduced muscle mass (TMESA/BSA $\leq 17.2 \mathrm{~cm}^{2} / \mathrm{m}^{2}$ ) and longer estimated waiting times, VAD implantation as a "bridge to transplantability" and specialized prehabilitation and nutrition programs to stop the progression of heart failure-associated cachexia, sarcopenia, and frailty should be considered as a possible treatment approach.

\section{Study Limitations}

This cohort represents the experience of a single center; therefore, it is not robust against systematic influencers caused by intrahospital standard procedures, such as the timing of emergency VAD implantation. In all our patients, long-term mechanical circulatory support with loss of the status "high urgent" was chosen, where possible, over stabilization with a short-term circulatory support like ECMO or Impella and continued listing in the high-urgency status, since the length of the waiting time is not predictable.

Patients were spared additional radiation in this retrospective analysis by using the standard CT for the evaluation; however, a

\section{REFERENCES}

1. Smits JM. Actual situation in Eurotransplant regarding high urgent heart transplantation. Eur J Cardiothorac Surg. (2012) 42:609-11. doi: 10.1093/ejcts/ezs424

2. Smits JM, de Vries E, De Pauw M, Zuckermann A, Rahmel A, Meiser $\mathrm{B}$, et al. Is it time for a cardiac allocation score? First results from the Eurotransplant pilot study on a survival benefit-based heart allocation. J Heart Lung Transplant. (2013) 32:873-80. doi: 10.1016/j.healun.2013.03.015 more standardized protocol including the exact timing of the CT and standardization of the contrast agent regimen might improve the significance and allow the measurement of muscle density as a potential surrogate for muscle quality.

According to our analysis, the impact of reduced muscle mass increases with the waiting time. Although this can be explained pathophysiologically, it warrants the support of prospective studies.

\section{CONCLUSIONS}

Muscle mass represented by TMESA/BSA at the time of listing for heart transplantation was a relevant risk factor for emergency VAD implantation or death during the $\mathrm{HU}$ waiting time prior to heart transplantation. Routine evaluation of muscle mass could identify patients at risk for decompensation during the waiting time and could help optimize the timing of VAD support.

\section{DATA AVAILABILITY STATEMENT}

The data analyzed in this study is subject to the following licenses/restrictions: Privacy policy for medical data. Requests to access these datasets should be directed to roehrich@dhzb.de.

\section{ETHICS STATEMENT}

The studies involving human participants were reviewed and approved by Ethics Committee of Charité-Universitätsmedizin Berlin (EA2/236/17). Written informed consent to participate in this study was provided by the participants' legal guardian/next of kin.

\section{AUTHOR CONTRIBUTIONS}

LR and FS administrative support and collection and assembly of data. LR, IJ, LK, NS, MH, JK, EP, CK, VF, and FS provision of study materials or patients. LR, SS, SK, JSt, and FS statistics, data analysis, and interpretation. All the authors concept and design, article draft, critical revision of article, and approval of article.

\section{FUNDING}

LR reports a research grant (Kaltenbach doctoral stipendium) from German Heart Foundation during the conduct of the study $(\mathrm{K} / 38 / 18)$. 
mortality. Am J Transplant. (2019) 19:2067-76. doi: 10.1111/ajt. 15265

5. Fried LP, Tangen CM, Walston J, Newman AB, Hirsch C, Gottdiener $\mathrm{J}$, et al. Cardiovascular health study collaborative research group. Frailty in older adults: evidence for a phenotype. J Gerontol A Biol Sci Med Sci. (2001) 56:M146-56. doi: 10.1093/gerona/56.3. M146

6. Cruz-Jentoft AJ, Bahat G, Bauer J, Boirie $\mathrm{Y}$, Bruyère $\mathrm{O}$, Cederholm T, et al. Writing group for the European Working Group on Sarcopenia in older people 2 (EWGSOP2), and the extended group for EWGSOP2. Sarcopenia: revised European consensus on definition and diagnosis. Age Ageing. (2019) 48:601. doi: 10.1093/ageing/afz046

7. McAdams-DeMarco MA, Ying H, Thomas AG, Warsame F, Shaffer AA, Haugen CE, et al. Frailty, inflammatory markers, and waitlist mortality among patients with end-stage renal disease in a prospective cohort study. Transplantation. (2018) 102:1740-6. doi: 10.1097/TP.0000000000 002213

8. Pérez Fernández M, Martínez Miguel P, Ying H, Haugen CE, Chu NM, Rodríguez Puyol DM, et al. Comorbidity, frailty, and waitlist mortality among kidney transplant candidates of all ages. Am J Nephrol. (2019) 49:103110. doi: $10.1159 / 000496061$

9. Montgomery E, Macdonald PS, Newton PJ, Chang S, Jha SR, Hannu MK, et al. Frailty as a predictor of mortality in patients with interstitial lung disease referred for lung transplantation. Transplantation. (2020) 104:86472. doi: $10.1097 /$ TP. 0000000000002901

10. Lai JC, Rahimi RS, Verna EC, Kappus MR, Dunn MA, McAdams-DeMarco $\mathrm{M}$, et al. Frailty associated with waitlist mortality independent of ascites and hepatic encephalopathy in a multicenter study. Gastroenterology. (2019) 156:1675-82. doi: 10.1053/j.gastro.2019.01.028

11. Haugen CE, McAdams-DeMarco M, Verna EC, Rahimi RS, Kappus MR, Dunn MA, et al. Association between liver transplant wait-list mortality and frailty based on body mass index. JAMA Surg. (2019) 154:11039. doi: 10.1001/jamasurg.2019.2845

12. Liu J, Yang BQ, Itoh A, Masood MF, Hartupee JC, Schilling JD. Impact of new unos allocation criteria on heart transplant practices and outcomes. Transplant Direct. (2020) 7:e642. doi: 10.1097/TXD.000000000 0001088

13. Organ Procurement and Transplantation Network. Adult Heart Allocation. (2018). Available online at: https://optn.transplant.hrsa. gov/learn/professional-education/adult-heart-allocation/ (accessed April 13, 2021).

14. Eurotransplant Manual Professionals. Chapter 6: ET Thoracic Allocation System (EThAS). (2021). Available online at: https://www.eurotransplant.org/ professionals/eurotransplant-manual/ (accessed February 20, 2021).

15. Minegishi Y, Inoue S, Sato K, Abe K, Murano H, Furuyama K, et al. Smaller erector spinae muscle size is associated with inability to recover activities of daily living after pneumonia treatment. Respir Investig. (2019) 57:1917. doi: 10.1016/j.resinv.2018.11.002

16. Miller JA, Harris K, Roche C, Dhillon S, Battoo A, Demmy T, et al. Sarcopenia is a predictor of outcomes after lobectomy. J Thorac Dis. (2018) 10:43240. doi: 10.21037/jtd.2017.12.39

17. Cruz-Jentoft AJ, Baeyens JP, Bauer JM, Boirie Y, Cederholm T, Landi F, et al. European working group on sarcopenia in older people. Sarcopenia: European consensus on definition and diagnosis: report of the European working group on sarcopenia in older people. Age Ageing. (2010) 39:41223. doi: 10.1093 /ageing/afq034

18. Wang FZ, Sun H, Zhou J, Sun LL, Pan SN. Reliability and validity of abdominal skeletal muscle area measurement using magnetic resonance imaging. Acad Radiol. (2020) 28:S1076-6332(20)30552-3. doi: 10.1016/j.acra.2020. 09.013

19. Tanimura K, Sato S, Fuseya Y, Hasegawa K, Uemasu K, Sato A, et al. Quantitative assessment of erector spinae muscles in patients with chronic obstructive pulmonary disease. Novel chest computed tomographyderived index for prognosis. Ann Am Thorac Soc. (2016) 13:33441. doi: 10.1513/AnnalsATS.201507-446OC

20. Oh J, Song IK, Nam JS, Lee SW, Lee EH, Choi IC. Sarcopenia as a prognostic factor for outcomes after isolated tricuspid valve surgery. J Cardiol. (2020) 76:585-92. doi: 10.1016/j.jjcc.2020.07.010
21. Heuman DM, Mihas AA, Habib A, Gilles HS, Stravitz RT, Sanyal AJ, et al. MELD-XI: a rational approach to "sickest first" liver transplantation in cirrhotic patients requiring anticoagulant therapy. Liver Transpl. (2007) 13:30-7. doi: 10.1002/lt.20906

22. Denfeld QE, Winters-Stone K, Mudd JO, Gelow JM, Kurdi S, Lee CS. The prevalence of frailty in heart failure: a systematic review and meta-analysis. Int J Cardiol. (2017) 236:283-9. doi: 10.1016/j.ijcard.2017.01.153

23. Vitale C, Jankowska E, Hill L, Piepoli M, Doehner W, Anker SD, et al. Heart Failure association/european society of cardiology position paper on frailty in patients with heart failure. Eur J Heart Fail. (2019) 21:1299305. doi: 10.1002/ejhf.1611

24. Smits JM, Deng MC, Hummel M, De Meester J, Schoendube F, Scheld HH, et al. Comparative outcome and clinical profiles in transplantation (COCPIT) study group. A prognostic model for predicting waiting-list mortality for a total national cohort of adult heart-transplant candidates. Transplantation. (2003) 76:1185-9. doi: 10.1097/01.TP.0000091171.82384.33

25. Leng SX, Kittleson MM. Beyond the eyeball test: impact and potential mechanisms of frailty in heart transplant candidates. J Heart Lung Transplant. (2021) 40:95-8. doi: 10.1016/j.healun.2020.12.004

26. Preoperative Nutritional Optimization and Physical Exercise for Patients Scheduled for Elective Implantation for a Left-Ventricular Assist Device (PROPER-LVAD). Available online at: https://clinicaltrials.gov/ct2/show/ NCT04205760?term=proper+VAD\&draw=1\&rank=1 (accessed April 30, 2021).

27. McCann M, Stamp N, Ngui A, Litton E. Cardiac Prehabilitation. J Cardiothorac Vasc Anesth. (2019) 33:2255-65. doi: 10.1053/j.jvca.2019. 01.023

28. Gimeno-Santos E, Coca-Martinez M, Arguis MJ, Navarro R, Lopez-Hernandez A, Castel MA, et al. Multimodal prehabilitation as a promising strategy for preventing physical deconditioning on the heart transplant waiting list. Eur J Prev Cardiol. (2020) 27:2367-70. doi: 10.1177/2047487319889709

29. Maurer MS, Horn E, Reyentovich A, Dickson VV, Pinney S, Goldwater $D$, et al. Can a left ventricular assist device in individuals with advanced systolic heart failure improve or reverse frailty? J Am Geriatr Soc. (2017) 65:2383-90. doi: 10.1111/jgs.15124

30. Jha SR, Hannu MK, Newton PJ, Wilhelm K, Hayward CS, Jabbour A, et al. Reversibility of frailty after bridge-to-transplant ventricular assist device implantation or heart transplantation. Transplant Direct. (2017) 3:e167. doi: 10.1097/TXD.0000000000000690

31. Adamopoulos S, Corrà U, Laoutaris ID, Pistono M, Agostoni PG, Coats AJS, et al. Exercise training in patients with ventricular assist devices: a review of the evidence and practical advice. A position paper from the committee on exercise physiology and training and the committee of advanced heart failure of the heart failure association of the european society of cardiology. Eur $J$ Heart Fail. (2019) 21:3-13. doi: 10.1002/ejhf.1352

32. International Thoracic Organ Transplant (TTX) Registry Data Slides. Adult Heart Transplantation Statistics. (2019). Available online at: https:// ishltregistries.org/registries/slides.asp?yearToDisplay=2019 (accessed February 20, 2021).

33. Belletti A, Jacobs S, Affronti G, Mladenow A, Landoni G, Falk V, et al. Incidence and predictors of postoperative need for high-dose inotropic support in patients undergoing cardiac surgery for infective endocarditis. J Cardiothorac Vasc Anesth. (2018) 32:2528-36. doi: 10.1053/j.jvca.2017. 12.015

Conflict of Interest: VF reports grants from Medtronic GmbH, Abbottt GmbH \& Co. KG, Boston Scientific, Edwards Lifesciences, JOTEC/CryoLife and other financial activities from Berlin Heart, Biotronik SE \& Co., Novartis Pharma $\mathrm{GmbH}$, Zurich Heart outside of the submitted work. FS reports other financial activities from Novartis, Abbott, Orion Pharma, Astra Zeneca and non-financial support from Medtronic outside of the submitted work. LR reports grants from German Heart Foundation during the conduct of the study, share holdings of Alianz SE, Carl Zeiss Meditec AG, CompuGroup Medical SE \& Co. KGaA, Evotec SE, Fresenius Medical Care AG \& Co. KGaA outside of the submitted work.

The remaining authors declare that the research was conducted in the absence of any commercial or financial 
relationships that could be construed as a potential conflict of interest.

Publisher's Note: All claims expressed in this article are solely those of the authors and do not necessarily represent those of their affiliated organizations, or those of the publisher, the editors and the reviewers. Any product that may be evaluated in this article, or claim that may be made by its manufacturer, is not guaranteed or endorsed by the publisher.
Copyright (C) 2021 Roehrich, Suendermann, Just, Kopp Fernandes, Schnettler, Kelle, Solowjowa, Stein, Hummel, Knierim, Potapov, Knosalla, Falk and Schoenrath. This is an open-access article distributed under the terms of the Creative Commons Attribution License (CC BY). The use, distribution or reproduction in other forums is permitted, provided the original author(s) and the copyright owner(s) are credited and that the original publication in this journal is cited, in accordance with accepted academic practice. No use, distribution or reproduction is permitted which does not comply with these terms. 